\title{
Third-Order Newton-Type Methods Combined with Vector Extrapolation for Solving Nonlinear Systems
}

\author{
Wen Zhou $^{1}$ and Jisheng Kou ${ }^{2}$ \\ ${ }^{1}$ Department of Foundation Courses, Hubei Vocational Technical College, Xiaogan, Hubei 432000, China \\ ${ }^{2}$ School of Mathematics and Statistics, Hubei Engineering University, Xiaogan, Hubei 432000, China \\ Correspondence should be addressed to Jisheng Kou; koujisheng@163.com
}

Received 22 March 2014; Accepted 31 May 2014; Published 15 June 2014

Academic Editor: Vinay Kanwar

Copyright (c) $2014 \mathrm{~W}$. Zhou and J. Kou. This is an open access article distributed under the Creative Commons Attribution License, which permits unrestricted use, distribution, and reproduction in any medium, provided the original work is properly cited.

We present a third-order method for solving the systems of nonlinear equations. This method is a Newton-type scheme with the vector extrapolation. We establish the local and semilocal convergence of this method. Numerical results show that the composite method is more robust and efficient than a number of Newton-type methods with the other vector extrapolations.

\section{Introduction}

Finding the solution of nonlinear equations is important in scientific and engineering computing areas. In this paper, we focus on the following nonlinear system of equations:

$$
F(x)=0
$$

where $F: \mathbb{R}^{n} \rightarrow \mathbb{R}^{n}$ is differentiable. Here, $F(x)=\left(f_{1}(x)\right.$, $\left.f_{2}(x), \ldots, f_{n}(x)\right)^{T}$ and $x \in \mathbb{R}^{n}$.

Some efficient methods for solving the system of (1) have been brought forward [1]. The Newton method for (1) is a second-order method. Its iterative formula is given by

$$
x_{k+1}=x_{k}-F^{\prime}\left(x_{k}\right)^{-1} F\left(x_{k}\right) \text {, }
$$

where $x_{k}$ is the current approximate solution and $F^{\prime}\left(x_{k}\right)$ is the Jacobian matrix of $F(x)$ at $x_{k}$. Potra and Pták [2] propose the modified Newton method (PPM) given by

$$
\begin{gathered}
y_{k}=x_{k}-F^{\prime}\left(x_{k}\right)^{-1} F\left(x_{k}\right), \\
x_{k+1}=y_{k}-F^{\prime}\left(x_{k}\right)^{-1} F\left(y_{k}\right) .
\end{gathered}
$$

In each iteration, PPM needs two evaluations of the vector function and one evaluation of the Jacobian matrix and the order is three.

Though the PPM can reduce the computational cost of Jacobian matrix, in some cases, the sequences produced by PPM converge slowly and even cannot converge because of the accumulation of the computational error. This problem limits its practical application.

In order to solve this problem, we will introduce the vector extrapolation technique to improve the convergence of PPM. Many vector extrapolation methods have been developed, such as the minimal polynomial extrapolation (MPE) method [3], the reduced rank extrapolation (RRE) method [4, $5]$, the modified minimal polynomial extrapolation (MMPE) method [6-8], the topological $\varepsilon$-algorithm (TEA) [6], and vector $\varepsilon$-algorithms (VEA) [9, 10]; also see [11, 12] and the references therein. These methods could be applied to the solvers of linear and nonlinear systems and accelerate their convergence.

In this paper, we construct a new extrapolation method and combine it with PPM, thus obtaining a Newton-type method. We will show by numerical results that the composite method can be of practical interest. The local and semilocal convergence are also established for the method. 


\section{The Method}

We introduce the following Newton-type method:

$$
\begin{gathered}
y_{k}=x_{k}-F^{\prime}\left(x_{k}\right)^{-1} F\left(x_{k}\right), \\
z_{k}=y_{k}-F^{\prime}\left(x_{k}\right)^{-1} F\left(y_{k}\right), \\
x_{k+1}=z_{k}-2 \frac{\left(x_{k}-y_{k}-\omega\left(y_{k}-z_{k}\right)\right) \cdot\left(z_{k}-y_{k}\right)}{\left\|x_{k}-y_{k}-\omega\left(y_{k}-z_{k}\right)\right\|^{2}}\left(z_{k}-y_{k}\right),
\end{gathered}
$$

where $\|\cdot\|$ is Euclidean norm and $0<\omega \leq 2$.

This iteration scheme consists of a PPM iterate to get $z_{k}$ from $x_{k}$, followed by a modified iterate to calculate $x_{k+1}$ from $x_{k}, y_{k}$, and $z_{k}$.

We now derive the last substep. Let $f(x)=0$ be a scalar real equation; then King's method [13] is described as

$$
\begin{gathered}
y_{k}=x_{k}-\frac{f\left(x_{k}\right)}{f^{\prime}\left(x_{k}\right)}, \\
z_{k}=y_{k}-\frac{f\left(y_{k}\right)}{f^{\prime}\left(x_{k}\right)}, \\
x_{k+1}=z_{k}-\frac{2\left(z_{k}-y_{k}\right)^{2}}{x_{k}-y_{k}-\omega\left(y_{k}-z_{k}\right)} .
\end{gathered}
$$

In order to extend the method (5) to the case of vector functions, we define the vector inverse as

$$
v^{-1}=\frac{v^{T}}{\|v\|^{2}}, \quad v \in \mathbb{R}^{n} .
$$

The last substep is obtained by applying the above vector inverse to the scalar King method.

The following theorem will give the order of convergence of the method with $0<\omega \leq 2$ given by (4).

Theorem 1. Suppose that the function $F: D \subset \mathbb{R}^{n} \rightarrow \mathbb{R}^{n}$ is continuously differentiable and $F^{\prime}\left(x^{*}\right)$ is nonsingular, where $D$ is an open set and $x^{*} \in D$ is the solution of $F(x)=0$. Define $\lambda=\left\|F^{\prime}\left(x^{*}\right)^{-1}\right\|$. Further, assume that there exists a positive number $\gamma$ such that for any $x \in D$,

$$
\left\|F^{\prime}(x)-F^{\prime}\left(x^{*}\right)\right\| \leq \gamma\left\|x-x^{*}\right\| ;
$$

then there exists a set $S$ such that for any $x_{0} \in S$, the sequence $\left\{x_{k}\right\}$ generated by (4) with $0<\omega \leq 2$ converges to $x^{*}$ and the order of convergence is three.

Proof. We can write (4) as $x_{k+1}=G\left(x_{k}\right)$ where

$$
\begin{gathered}
y=x-F^{\prime}(x)^{-1} F(x), \quad z=y-F^{\prime}(x)^{-1} F(y), \\
G(x)=z-2 \frac{(x-y-\omega(y-z)) \cdot(z-y)}{\|x-y-\omega(y-z)\|^{2}}(z-y) .
\end{gathered}
$$

Without loss of generality, we use the Euclidean norms as $\|\cdot\|$ in the following. Let $\delta=1 / 20 \lambda \gamma$ and $S=\left\{x \mid\left\|x-x^{*}\right\| \leq\right.$ $\delta\} \cap D$. Let $x \in S$ and $x \neq x^{*}$.
It is obtained from (7) that

$$
\left\|F^{\prime}(x)-F^{\prime}\left(x^{*}\right)\right\| \leq \gamma \delta \leq \frac{1}{20 \lambda} .
$$

By Banach lemma, we obtain that $F^{\prime}(x)$ is nonsingular and $\left\|F^{\prime}(x)^{-1}\right\| \leq(20 / 19) \lambda$. So $y$ and $z$ are well defined.

By making use of Taylor expansion and (7), we have

$$
\begin{aligned}
& \left\|F\left(x^{*}\right)-F(x)-F^{\prime}(x)\left(x^{*}-x\right)\right\| \\
& =\left\|\int_{0}^{1} F^{\prime}\left(x+t\left(x^{*}-x\right)\right)\left(x^{*}-x\right) d t-F^{\prime}(x)\left(x^{*}-x\right)\right\| \\
& =\left\|\int_{0}^{1}\left[F^{\prime}\left(x+t\left(x^{*}-x\right)\right)-F^{\prime}(x)\right]\left(x^{*}-x\right) d t\right\| \\
& \leq \int_{0}^{1}\left\|F^{\prime}\left(x+t\left(x^{*}-x\right)\right)-F^{\prime}(x)\right\|\left\|x^{*}-x\right\| d t \\
& \leq \gamma\left\|x-x^{*}\right\|^{2} \int_{0}^{1} t d t \\
& =\frac{1}{2} \gamma\left\|x-x^{*}\right\|^{2} .
\end{aligned}
$$

So for $y$ we obtain

$$
\begin{aligned}
\left\|y-x^{*}\right\| & =\left\|F^{\prime}(x)^{-1}\right\|\left\|F\left(x^{*}\right)-F(x)-F^{\prime}(x)\left(x^{*}-x\right)\right\| \\
& \leq \frac{10}{19} \lambda \gamma\left\|x-x^{*}\right\|^{2} .
\end{aligned}
$$

Similarly to (10), we get

$$
\left\|F(y)-F\left(x^{*}\right)-F^{\prime}\left(x^{*}\right)\left(y-x^{*}\right)\right\| \leq \frac{1}{2} \gamma\left\|y-x^{*}\right\|^{2} .
$$

It is obtained by (7) and (12) that

$$
\begin{aligned}
& \left\|z-x^{*}\right\| \\
& \leq\left\|F^{\prime}(x)^{-1}\right\|\left[\left\|F^{\prime}(x)-F^{\prime}\left(x^{*}\right)\right\|\left\|y-x^{*}\right\|\right. \\
& \left.+\left\|F\left(x^{*}\right)-F(y)-F^{\prime}\left(x^{*}\right)\left(x^{*}-y\right)\right\|\right] \\
& \leq \frac{200}{361} \lambda^{2} \gamma^{2}\left\|x-x^{*}\right\|^{3}+\frac{1000}{6859} \lambda^{3} \gamma^{3}\left\|x-x^{*}\right\|^{4} .
\end{aligned}
$$


Therefore it follows that

$$
\begin{aligned}
\| G(x)- & x^{*} \| \\
\leq & \left(\left\|x-x^{*}\right\|\left\|z-x^{*}\right\|+(3-\omega)\left\|z-x^{*}\right\|\left\|y-x^{*}\right\|\right. \\
& \left.\quad+(2-\omega)\left\|z-x^{*}\right\|^{2}+2\left\|y-x^{*}\right\|^{2}\right) \\
& \times\left(\left\|x-x^{*}\right\|-(\omega+1)\left\|y-x^{*}\right\|-\omega\left\|z-x^{*}\right\|\right)^{-1} \\
\leq & \frac{292700}{480111} \lambda^{2} \gamma^{2}\left\|x-x^{*}\right\|^{3} \\
\leq & \frac{2927}{1920444}\left\|x-x^{*}\right\|<\left\|x-x^{*}\right\| .
\end{aligned}
$$

This proves that $G(x) \in S$ and $G$ is a contraction mapping. Thus, for any $x_{0} \in S$, the sequence $\left\{x_{k}\right\}$ produced by (4) is well defined and it converges to $x^{*}$. Finally, it is shown from (13) that the order of the method (4) is three.

\section{The Semilocal Convergence}

In this section, we will establish the semilocal convergence of method (4). This convergence may be derived by using recurrence relations, which have been used in establishing the convergence of Newton's method and some third-order methods [14-29]. In what follows, an attempt is made to use recurrence relations to establish the semilocal convergence for the method (4). The recurrence relations based on one constant which depend on $F$ are derived. Further, based on these recurrence relations, the error estimate is obtained for the present iterative method.

In order to establish the recurrence relations for the present iterative method, we will use the following scalar functions which are defined by

$$
\begin{aligned}
& g_{1}(t)=1+\frac{1}{2} t+\frac{t^{2}}{2-\omega t}, \\
& g_{2}(t)=\frac{1}{1-\operatorname{tg}_{1}(t)}, \\
& g_{3}(t)=\frac{1}{2} t^{2} g_{2}(t)\left[\frac{4+(2-\omega) t}{2-\omega t}+\frac{1}{4} t\left(\frac{2+(2-\omega) t}{2-\omega t}\right)^{2}\right], \\
& g_{4}(t)=g_{2}(t) g_{3}(t),
\end{aligned}
$$

where $0<\omega \leq 2$.

Let $h(t)=(2-\omega t)\left(\operatorname{tg}_{1}(t)-1\right)=(1-(1 / 2) \omega) t^{3}+(1-$ $\omega) t^{2}+(2+w) t-2$. For any positive real number $\omega$, it is easy to obtain $h(0) h(2 / \omega)<0$, so $h(t)$ has at least a real zero point $\widehat{t} \epsilon$ $(0,2 / \omega)$. Furthermore, let $f(t)=g_{4}(t)-1$. It can be included $f(0)<0$ and $f(t) \rightarrow+\infty$, so $f(t)$ has at least a real zero in $t^{*} \in(0, \hat{t})$. Furthermore, it can be obtained that $f(t)$ is an increasing function in $(0,2 / \omega)$. So $t^{*}$ is the unique zero of $f(t)$ in $(0,2 / \omega)$. For the functions defined by $(15)$, we have the following results.
Lemma 2. Let $t^{*}$ be the unique real root of $g_{4}(t)-1=0$ in $(0,2 / \omega)$. Then

(a) $g_{1}(t)$ is an increasing function in $\left[0, t^{*}\right]$ and satisfies $1 \leq g_{1}(t) \leq g_{1}\left(t^{*}\right)$

(b) $g_{2}(t)$ is an increasing function in $\left[0, t^{*}\right]$ and satisfies $1 \leq g_{2}(t) \leq g_{2}\left(t^{*}\right)$

(c) $g_{3}(t)$ is an increasing function in $\left[0, t^{*}\right]$ and satisfies $0 \leq g_{3}(t) \leq g_{3}\left(t^{*}\right)<1$

(d) $g_{4}(t)$ is an increasing function in $\left[0, t^{*}\right]$ and satisfies $0 \leq g_{4}(t) \leq g_{4}\left(t^{*}\right)=1 ;$

(e) $t^{*} g_{1}\left(t^{*}\right) /\left(1-g_{3}\left(t^{*}\right)\right)=1$.

Proof. The results (a)-(d) can be obtained by simple derivations. We only prove the validity of (e). Noticing that

$$
\begin{aligned}
& g_{4}(t)=g_{2}(t) g_{3}(t), \\
& g_{2}(t)=\frac{1}{1-\operatorname{tg}_{1}(t)},
\end{aligned}
$$

we get

$$
1=g_{4}\left(t^{*}\right)=\frac{g_{3}\left(t^{*}\right)}{1-t^{*} g_{1}\left(t^{*}\right)},
$$

which can be converted to (e).

Theorem 3. Assume that the function $F: D \subset \mathbb{R}^{n} \rightarrow \mathbb{R}^{n}$ is continuously differentiable where $D$ is an open set and there exists a positive number $\gamma$ such that for any $x, v \in D$

$$
\left\|F^{\prime}(x)-F^{\prime}(v)\right\| \leq \gamma\|x-v\| .
$$

Let $g_{1}(t), g_{2}(t), g_{3}(t)$, and $g_{4}(t)$ be defined by (15). Further, define $\alpha_{k}, \beta_{k}$, and $\rho_{k}$ as

$$
\begin{gathered}
\alpha_{k}=\left\|F^{\prime}\left(x_{k}\right)^{-1} F\left(x_{k}\right)\right\|, \quad \beta_{k}=\left\|F^{\prime}\left(x_{k}\right)^{-1}\right\|, \\
\rho_{k}=\alpha_{k} \beta_{k} \gamma .
\end{gathered}
$$

Let $x_{0} \in D$ satisfy $F\left(x_{0}\right) \neq 0, F^{\prime}\left(x_{0}\right)$ be nonsingular, $\rho_{0} \in$ $\left(0, t^{*}\right) \subset(0,2 / \omega)$, and $S=\left\{x \mid\left\|x-x_{0}\right\| \leq \theta \alpha_{0}\right\} \subset D$ where $\theta=g_{1}\left(t^{*}\right) /\left(1-g_{3}\left(t^{*}\right)\right)$ and $t^{*}$ is the root of $g_{4}(t)-1=0$ in $(0,2 / \omega)$; then we have that

(i) $\left\{x_{k}\right\}$ generated by the method (4) is well defined in $S$ and satisfies

$$
\left\|x_{k+1}-x_{k}\right\| \leq g_{1}\left(\rho_{k}\right) \alpha_{k}
$$

(ii) $\alpha_{k}, \beta_{k}$, and $\rho_{k}$ are well defined and satisfy

$$
\begin{aligned}
& \alpha_{k+1} \leq g_{3}\left(\rho_{k}\right) \alpha_{k}, \\
& \beta_{k+1} \leq g_{2}\left(\rho_{k}\right) \beta_{k}, \\
& \rho_{k+1} \leq g_{4}\left(\rho_{k}\right) \rho_{k} .
\end{aligned}
$$


Proof. Without loss of generality, we use the Euclidean norms as $\|\cdot\|$ in the following. We firstly consider the case $k=0$.

Since $1<\theta$, it is obvious that $y_{0} \in S$ by the definition $\alpha_{0}=\left\|y_{0}-x_{0}\right\|$. By Taylor expansion we have

$$
\begin{aligned}
\| F & \left(y_{0}\right) \| \\
& =\left\|F\left(x_{0}\right)+F^{\prime}\left(x_{0}\right)\left(y_{0}-x_{0}\right)+\int_{x_{0}}^{y_{0}}\left[F^{\prime}(x)-F^{\prime}\left(x_{0}\right)\right] d x\right\| \\
& =\left\|\int_{0}^{1}\left[F^{\prime}\left(x_{0}+t\left(y_{0}-x_{0}\right)\right)-F^{\prime}\left(x_{0}\right)\right]\left(y_{0}-x_{0}\right) d t\right\| \\
& \leq \int_{0}^{1}\left\|F^{\prime}\left(x_{0}+t\left(y_{0}-x_{0}\right)\right)-F^{\prime}\left(x_{0}\right)\right\|\left\|y_{0}-x_{0}\right\| d t \\
& \leq \gamma\left\|y_{0}-x_{0}\right\|^{2} \int_{0}^{1} t d t \\
& =\frac{1}{2} \gamma \alpha_{0}^{2} .
\end{aligned}
$$

Furthermore,

$$
\left\|z_{0}-y_{0}\right\| \leq\left\|F^{\prime}\left(x_{0}\right)^{-1}\right\|\left\|F\left(y_{0}\right)\right\| \leq \frac{1}{2} \beta_{0} \gamma \alpha_{0}^{2}=\frac{1}{2} \rho_{0} \alpha_{0} .
$$

It then follows that

$$
\left\|z_{0}-x_{0}\right\| \leq\left\|z_{0}-y_{0}\right\|+\left\|y_{0}-x_{0}\right\| \leq\left(1+\frac{1}{2} \rho_{0}\right) \alpha_{0} .
$$

Taking account of the relation

$$
1+\frac{1}{2} \rho_{0}<g_{1}\left(\rho_{0}\right)<g_{1}\left(t^{*}\right)<\theta
$$

we have $z_{0} \in S$. Since

$$
\begin{aligned}
\left\|x_{0}-y_{0}-\omega\left(y_{0}-z_{0}\right)\right\| & \geq\left\|y_{0}-x_{0}\right\|-\omega\left\|z_{0}-y_{0}\right\| \\
& \geq\left(1-\frac{1}{2} \omega \rho_{0}\right) \alpha_{0}>0,
\end{aligned}
$$

we obtain that $x_{1}$ is well defined and

$$
\begin{aligned}
\left\|x_{1}-z_{0}\right\| & \leq \frac{2\left\|z_{0}-y_{0}\right\|^{2}}{\left\|x_{0}-y_{0}-\omega\left(y_{0}-z_{0}\right)\right\|} \\
& \leq \frac{\rho_{0}^{2} \alpha_{0}}{2-\omega \rho_{0}}, \\
\left\|x_{1}-y_{0}\right\| \leq\left\|x_{1}-z_{0}\right\|+\left\|z_{0}-y_{0}\right\| \leq \frac{\rho_{0} \alpha_{0}}{2} \frac{2+(2-\omega) \rho_{0}}{2-\omega \rho_{0}} & \\
\left\|x_{1}-x_{0}\right\| & \leq\left\|x_{1}-y_{0}\right\|+\left\|y_{0}-x_{0}\right\| \\
& \leq \alpha_{0}\left[1+\frac{1}{2} \rho_{0}+\frac{\rho_{0}^{2}}{2-\omega \rho_{0}}\right] \\
& =g_{1}\left(\rho_{0}\right) \alpha_{0}<\theta \alpha_{0} .
\end{aligned}
$$

This shows $x_{1} \in S$ and the validity of (20).
By condition (18) we have

$$
\left\|F^{\prime}\left(x_{1}\right)-F^{\prime}\left(x_{0}\right)\right\| \leq \gamma\left\|x_{1}-x_{0}\right\| \leq \gamma g_{1}\left(\rho_{0}\right) \alpha_{0} .
$$

Because

$$
\gamma g_{1}\left(\rho_{0}\right) \alpha_{0} \beta_{0}=g_{1}\left(\rho_{0}\right) \rho_{0}<1,
$$

by Banach lemma we obtain that $F^{\prime}\left(x_{1}\right)$ is nonsingular and

$$
\beta_{1}=\left\|F^{\prime}\left(x_{1}\right)^{-1}\right\| \leq \frac{\beta_{0}}{1-g_{1}\left(\rho_{0}\right) \rho_{0}}=g_{2}\left(\rho_{0}\right) \beta_{0} .
$$

This is to say that (22) holds.

Now we consider $F\left(x_{1}\right)$. By making use of (24), (25), and (30), we obtain

$\left\|F\left(x_{1}\right)\right\|$

$$
\begin{gathered}
=\| F\left(x_{0}\right)+F^{\prime}\left(x_{0}\right)\left(x_{1}-x_{0}\right) \\
+\int_{x_{0}}^{x_{1}}\left[F^{\prime}(x)-F^{\prime}\left(x_{0}\right)\right] d x \|
\end{gathered}
$$

$$
\begin{aligned}
\leq & \frac{2\left\|z_{0}-y_{0}\right\|}{\left\|x_{0}-y_{0}-\omega\left(y_{0}-z_{0}\right)\right\|}\left\|F\left(y_{0}\right)\right\| \\
& +\left\|-F\left(y_{0}\right)+\int_{x_{0}}^{x_{1}}\left[F^{\prime}(x)-F^{\prime}\left(x_{0}\right)\right] d x\right\|
\end{aligned}
$$$$
\leq \frac{2\left\|z_{0}-y_{0}\right\|\left\|F\left(y_{0}\right)\right\|}{\left\|y_{0}-x_{0}\right\|-\omega\left\|z_{0}-y_{0}\right\|}
$$$$
+\|-\int_{x_{0}}^{y_{0}}\left[F^{\prime}(x)-F^{\prime}\left(x_{0}\right)\right] d x
$$$$
+\int_{x_{0}}^{x_{1}}\left[F^{\prime}(x)-F^{\prime}\left(x_{0}\right)\right] d x \|
$$$$
=\frac{\gamma \rho_{0} \alpha_{0}^{2}}{2-\omega \rho_{0}}+\left\|\int_{y_{0}}^{x_{1}}\left[F^{\prime}(x)-F^{\prime}\left(x_{0}\right)\right] d x\right\|
$$$$
\leq \frac{\gamma \rho_{0} \alpha_{0}^{2}}{2-\omega \rho_{0}}+\int_{0}^{1}\left\|F^{\prime}\left(y_{0}+t\left(x_{1}-y_{0}\right)\right)-F^{\prime}\left(x_{0}\right)\right\|
$$

$$
\times\left\|x_{1}-y_{0}\right\| d t
$$




$$
\begin{aligned}
\leq & \frac{\gamma \rho_{0} \alpha_{0}^{2}}{2-\omega \rho_{0}} \\
& +\gamma \int_{0}^{1}\left[\left\|y_{0}-x_{0}\right\|+t\left\|x_{1}-y_{0}\right\|\right]\left\|x_{1}-y_{0}\right\| d t \\
\leq & \frac{\gamma \rho_{0} \alpha_{0}^{2}}{2-\omega \rho_{0}} \\
& +\gamma\left[\left\|y_{0}-x_{0}\right\|+\frac{1}{2}\left\|x_{1}-y_{0}\right\|\right]\left\|x_{1}-y_{0}\right\| \\
\leq & \frac{\gamma \rho_{0} \alpha_{0}^{2}}{2-\omega \rho_{0}} \\
& +\frac{\gamma}{2} \rho_{0} \alpha_{0}^{2} \frac{2+(2-\omega) \rho_{0}}{2-\omega \rho_{0}}\left[1+\frac{1}{4} \rho_{0} \frac{2+(2-\omega) \rho_{0}}{2-\omega \rho_{0}}\right] \\
= & \frac{\gamma}{2} \rho_{0} \alpha_{0}^{2}\left[\frac{4+(2-\omega) \rho_{0}}{2-\omega \rho_{0}}+\frac{1}{4} \rho_{0}\left(\frac{2+(2-\omega) \rho_{0}}{2-\omega \rho_{0}}\right)^{2}\right] .
\end{aligned}
$$

Finally, we prove (21) and (23). By making use of (34) and (35), we have

$$
\begin{aligned}
\alpha_{1} \leq & \left\|F^{\prime}\left(x_{1}\right)^{-1}\right\|\left\|F\left(x_{1}\right)\right\| \\
\leq & \frac{1}{2} \alpha_{0} \rho_{0}^{2} g_{2}\left(\rho_{0}\right) \\
& \times\left[\frac{4+(2-\omega) \rho_{0}}{2-\omega \rho_{0}}+\frac{1}{4} \rho_{0}\left(\frac{2+(2-\omega) \rho_{0}}{2-\omega \rho_{0}}\right)^{2}\right] \\
= & g_{3}\left(\rho_{0}\right) \alpha_{0} .
\end{aligned}
$$

It then holds that

$$
\begin{aligned}
\rho_{1} & =\alpha_{1} \beta_{1} \gamma \\
& \leq \rho_{0} g_{2}\left(\rho_{0}\right) g_{3}\left(\rho_{0}\right) \\
& =g_{4}\left(\rho_{0}\right) \rho_{0} \\
& <g_{4}\left(t^{*}\right) \rho_{0}=\rho_{0}<t^{*} .
\end{aligned}
$$

Now we consider the cases $k \geq 1$. By induction we can obtain the following facts.

(P1) By Lemma 2, we obtain that

$$
\rho_{k} \leq g_{4}\left(\rho_{k-1}\right) \rho_{k-1}<g_{4}\left(t^{*}\right) \rho_{k-1}=\rho_{k-1}<t^{*}
$$

which leads to

$$
\begin{gathered}
g_{3}\left(\rho_{k}\right)<g_{3}\left(\rho_{k-1}\right)<\cdots<g_{3}\left(\rho_{0}\right)<g_{3}\left(t^{*}\right)<1, \\
g_{1}\left(\rho_{k}\right)<g_{1}\left(t^{*}\right) .
\end{gathered}
$$

It follows that

$$
\alpha_{k} \leq g_{3}\left(\rho_{k-1}\right) \alpha_{k-1} \leq g_{3}\left(\rho_{k-1}\right) \cdots g_{3}\left(\rho_{0}\right) \alpha_{0}<g_{3}\left(t^{*}\right)^{k} \alpha_{0} .
$$

This further yields

$$
\left\|x_{k}-x_{k-1}\right\| \leq g_{1}\left(\rho_{k-1}\right) \alpha_{k-1}<g_{1}\left(t^{*}\right) g_{3}\left(t^{*}\right)^{k-1} \alpha_{0} .
$$

Thus it is obtained that

$$
\begin{aligned}
\left\|x_{k}-x_{0}\right\| & \leq \sum_{i=1}^{k}\left\|x_{i}-x_{i-1}\right\| \\
& <\sum_{i=1}^{k} g_{1}\left(t^{*}\right) g_{3}\left(t^{*}\right)^{i-1} \alpha_{0} .
\end{aligned}
$$

Next we show that $y_{k}, z_{k}$ are well defined in $S$. By Lemma 2 and (42), we have

$$
\begin{aligned}
\left\|y_{k}-x_{0}\right\| & \leq \alpha_{k}+\left\|x_{k}-x_{0}\right\| \\
& \leq g_{3}\left(t^{*}\right)^{k} \alpha_{0}+\sum_{i=1}^{k} g_{1}\left(t^{*}\right) g_{3}\left(t^{*}\right)^{i-1} \alpha_{0} \\
& <\sum_{i=0}^{k} g_{1}\left(t^{*}\right) g_{3}\left(t^{*}\right)^{i} \alpha_{0} \\
& <\frac{g_{1}\left(t^{*}\right)}{1-g_{3}\left(t^{*}\right)} \alpha_{0} .
\end{aligned}
$$

This means that $y_{k} \in S$. Furthermore, by analogous procedures to (24), (25), and (26), we obtain that

$$
\begin{gathered}
\left\|z_{k}-y_{k}\right\| \leq \frac{1}{2} \rho_{k} \alpha_{k}, \\
\left\|z_{k}-x_{k}\right\| \leq\left\|z_{k}-y_{k}\right\|+\left\|y_{k}-x_{k}\right\| \leq\left(1+\frac{1}{2} \rho_{k}\right) \alpha_{k} .
\end{gathered}
$$

Since

$$
1+\frac{1}{2} \rho_{k}<g_{1}\left(\rho_{k}\right)<g_{1}\left(t^{*}\right)
$$

we get

$$
\left\|z_{k}-x_{k}\right\|<g_{1}\left(\rho_{k}\right) \alpha_{k}<g_{1}\left(t^{*}\right) g_{3}\left(t^{*}\right)^{k} \alpha_{0} .
$$

Hence it follows that

$$
\begin{aligned}
\left\|z_{k}-x_{0}\right\| & \leq\left\|z_{k}-x_{k}\right\|+\left\|x_{k}-x_{0}\right\| \\
& \leq \sum_{i=0}^{k} g_{1}\left(t^{*}\right) g_{3}\left(t^{*}\right)^{i} \alpha_{0} \\
& <\frac{g_{1}\left(t^{*}\right)}{1-g_{3}\left(t^{*}\right)} \alpha_{0} .
\end{aligned}
$$

This shows that $z_{k} \in S$. Similarly to the case $k=0$, we obtain that $x_{k+1}$ is well defined and have

$$
\begin{gathered}
\left\|x_{k+1}-z_{k}\right\| \leq \frac{\rho_{k}^{2} \alpha_{k}}{2-\omega \rho_{k}}, \\
\left\|x_{k+1}-y_{k}\right\| \leq\left\|x_{k+1}-z_{k}\right\|+\left\|z_{k}-y_{k}\right\| \leq \frac{\rho_{k} \alpha_{k}}{2} \frac{2+(2-\omega) \rho_{k}}{2-\omega \rho_{k}}, \\
\left\|x_{k+1}-x_{k}\right\| \leq g_{1}\left(\rho_{k}\right) \alpha_{k} .
\end{gathered}
$$


By (42) we obtain

$$
\left\|x_{k+1}-x_{0}\right\|<\frac{g_{1}\left(t^{*}\right)}{1-g_{3}\left(t^{*}\right)} \alpha_{0},
$$

which shows $x_{k+1} \in S$.

(P2) We can prove analogously to (35) that

$$
\begin{aligned}
& \left\|F\left(x_{k+1}\right)\right\| \\
& \quad \leq \frac{\gamma}{2} \rho_{k} \alpha_{k}^{2}\left[\frac{4+(2-\omega) \rho_{k}}{2-\omega \rho_{k}}+\frac{1}{4} \rho_{k}\left(\frac{2+(2-\omega) \rho_{k}}{2-\omega \rho_{k}}\right)^{2}\right] .
\end{aligned}
$$

(P3) Because

$$
\begin{gathered}
\left\|F^{\prime}\left(x_{k+1}\right)-F^{\prime}\left(x_{k}\right)\right\| \leq \gamma g_{1}\left(\rho_{k}\right) \alpha_{k}, \\
g_{1}\left(\rho_{k}\right) \rho_{k}<g_{1}\left(\rho_{0}\right) \rho_{0}<1,
\end{gathered}
$$

we obtain that $F^{\prime}\left(x_{k+1}\right)$ is nonsingular and

$$
\beta_{k+1}=\left\|F^{\prime}\left(x_{k+1}\right)^{-1}\right\| \leq g_{2}\left(\rho_{k}\right) \beta_{k} .
$$

(P4) From (50) and (52), we have

$$
\alpha_{k+1} \leq g_{3}\left(\rho_{k}\right) \alpha_{k}
$$

It then follows that

$$
\begin{aligned}
\rho_{k+1} & =\alpha_{k+1} \beta_{k+1} \gamma \\
& \leq g_{4}\left(\rho_{k}\right) \rho_{k} \\
& <g_{4}\left(t^{*}\right) \rho_{k}=\rho_{k} .
\end{aligned}
$$

Thus far, we have proved all conclusions of this theorem.

The theorem given below will establish the convergence of the sequence $\left\{x_{k}\right\}$ and give the error estimate for it.

Theorem 4. Let the conditions of Theorem 3 be satisfied. Denote $p=g_{1}\left(t^{*}\right)$ and $q=g_{3}\left(t^{*}\right)$. Then the sequence $\left\{x_{k}\right\}$ generated by (4) converges to a unique solution $x^{*} \in S$ of $F(x)$, and it holds that

$$
\left\|x^{*}-x_{k}\right\|<\frac{p q^{k}}{1-q} \alpha_{0} .
$$

Proof. Since $0<q<1$, it follows from (41) that

$$
\begin{aligned}
\left\|x_{k+m}-x_{k}\right\| & \leq \sum_{i=0}^{m-1}\left\|x_{k+i+1}-x_{k+i}\right\| \\
& <\sum_{i=0}^{m-1} p q^{k+i} \alpha_{0} \\
& <\frac{p q^{k}}{1-q} \alpha_{0} .
\end{aligned}
$$

This means that $\left\{x_{k}\right\}$ is a Cauchy sequence and thus there exists a $x^{*}$ such that $\lim _{k \rightarrow \infty} x_{k}=x^{*}$. By letting $m \rightarrow \infty$ in (56), we obtain (55). From (56) and (42), we can get

$$
\begin{aligned}
\left\|x^{*}-x_{0}\right\| & \leq\left\|x^{*}-x_{k}\right\|+\left\|x_{k}-x_{0}\right\| \\
& <\sum_{i=0}^{\infty} p q^{i} \alpha_{0}=\frac{p}{1-q} \alpha_{0} .
\end{aligned}
$$

This shows $x^{*} \in S$.

From (50) and (40), we obtain that

$$
\begin{aligned}
\left\|F\left(x_{k}\right)\right\|< & \frac{\gamma}{2} \rho_{0} \alpha_{0}^{2} q^{2(k-1)} \\
& \times\left[\frac{4+(2-\omega) \rho_{0}}{2-\omega \rho_{0}}+\frac{1}{4} \rho_{0}\left(\frac{2+(2-\omega) \rho_{0}}{2-\omega \rho_{0}}\right)^{2}\right] .
\end{aligned}
$$

By letting $k \rightarrow \infty$ in (58), we obtain $F\left(x^{*}\right)=0$; namely, $x^{*}$ is a solution of $F(x)$.

Now, we prove the uniqueness of $x^{*}$ in $S$. Let $x^{* *}$ be another zero of $F(x)$ in $S$. By mean value theorem, we have

$$
0=F\left(x^{* *}\right)-F\left(x^{*}\right)=F^{\prime}(\xi)\left(x^{* *}-x^{*}\right),
$$

where $\xi$ is between $x^{*}$ and $x^{* *}$. Since

$$
\begin{aligned}
\beta_{0}\left\|F^{\prime}(\xi)-F^{\prime}\left(x_{0}\right)\right\| & \leq \beta_{0} \gamma\left\|\xi-x_{0}\right\| \\
& \leq \rho_{0} \frac{g_{1}\left(t^{*}\right)}{1-g_{3}\left(t^{*}\right)} \\
& <\frac{t^{*} g_{1}\left(t^{*}\right)}{1-g_{3}\left(t^{*}\right)}=1,
\end{aligned}
$$

it follows by Banach lemma that $F^{\prime}(\xi)$ is invertible and hence $x^{* *}=x^{*}$. This ends the proof.

\section{Numerical Tests}

In this section, we present some numerical results for the method given by (4) (NTM) and compare it with PPM on their numerical behavior. We also test the composite methods combining PPM with some known vector extrapolation methods mentioned in Section 1, which are indicated as VEAPPM, MPE-PPM, and RRE-PPM, respectively. We use $\left\|F_{k}\right\|_{2}$ to denote the value of $\|F(x)\|_{2}$ at the $k$ th approximate solution $x_{k}$.

We consider the nonlinear elliptic differential equation:

$$
-\nabla \cdot K(\Theta(\psi)) \nabla \psi=0 .
$$

This equation often arises from the flow model in porous media and in this case, $\psi$ is the pressure, $\Theta$ the fluid saturation, and $K$ the conductivity. The boundary conditions can be given by

$$
\begin{gathered}
-K(\Theta(\psi)) \nabla \psi=V_{B}, \quad \text { on } \Gamma_{N}, \\
\psi=\psi_{B}, \quad \text { on } \Gamma_{D} .
\end{gathered}
$$


TABLE 1: Results of the case $m=100$.

\begin{tabular}{lccccc}
\hline$x_{0}$ & PPM & VEA-PPM & MPE-PPM & RRE-PPM & NTM \\
\hline $0.5 S$ & D & D & 8 & 8 & 5 \\
$1 S$ & D & 6 & 7 & 7 & 5 \\
$1.5 S$ & 22 & 6 & 6 & 6 & 5 \\
$2 S$ & D & 5 & 6 & 6 & 4 \\
$3 S$ & D & 5 & 6 & 6 & 4 \\
\hline
\end{tabular}

TABLE 2: Results of the case $m=1000$.

\begin{tabular}{lccccc}
\hline$x_{0}$ & PPM & VEA-PPM & MPE-PPM & RRE-PPM & NTM \\
\hline $0.1 S$ & D & D & 10 & 10 & 7 \\
$0.5 S$ & D & D & 9 & 9 & 6 \\
$1 S$ & D & 30 & 8 & 8 & 6 \\
$1.5 S$ & $\mathrm{D}$ & 8 & 7 & 7 & 5 \\
$2 S$ & $\mathrm{D}$ & 7 & 7 & 7 & 5 \\
$3 S$ & $\mathrm{D}$ & 6 & 7 & 7 & 5 \\
\hline
\end{tabular}

In this test, we consider the one-dimensional case. The uniform cell-centered finite difference (CCFD) approximation method is used to discretize the boundary value problem. For the detailed CCFD formulations, we refer to [30] or the references therein. The values $K(\Theta(\psi))$ on the faces of each cell are taken as the harmonic mean of cell-central ones. Here, we take $K(\Theta(\psi))=k \psi$ where $k$ is a positive real constant. The input boundary condition is given by $V_{B}=1$, while the output boundary condition is $\psi_{B}=1$.

The discrete scheme leads to a nonlinear equation system with $m$ variables. We test two cases with the sizes $m=100$ and 1000 , respectively. We take $\omega=2$ in our method. All methods start from the initial approximate solutions and stop when they satisfy the given criteria. For the case $m=100$, the stopping criterion is $\|F\|_{2}<1 e-12$, while it is taken as $\|F\|_{2}<1 e-11$ for $m=1000$. In these tables, we show the iteration number cost by various methods.

The computational results are displayed in Tables 1 and 2. In the tables, denote $S=(1,1, \ldots, 1)^{T}$ and " $D$ " indicates that the method is divergent or cannot converge in 50 steps. We use NTM to represent the proposed method.

From the numerical results, we can know that the performance of NTM is more efficient and robust than PPM.

\section{Conclusions}

We establish the convergence of a third-order method for systems of nonlinear equations; an existence-uniqueness theorem and the error estimate for this method are also obtained. Numerical results show that this method is more robust and efficient than a number of Newton-type methods with the other vector extrapolation algorithms.

\section{Conflict of Interests}

The authors declare that there is no conflict of interests regarding the publication of this paper.

\section{Acknowledgment}

This work is supported by the Scientific and Technical Research Project of Hubei Provincial Department of Education (no. D20132701).

\section{References}

[1] J. M. Ortega and W. C. Rheinboldt, Iterative Solution of Nonlinear Equations in Several Variables, Academic Press, New York, NY, USA, 1970.

[2] F. A. Potra and V. Pták, Nondiscrete Induction and Iterative Processes, vol. 103 of Research Notes in Mathematics, Pitman, Boston, Mass, USA, 1984.

[3] S. Cabay and L. W. Jackson, "A polynomial extrapolation method for finding limits and antilimits of vector sequences," SIAM Journal on Numerical Analysis, vol. 13, no. 5, pp. 734-752, 1976.

[4] R. P. Eddy, "Extrapolation to the limit of a vector sequence," in Information Linkage Between Applied Mathematics and Industry, P. C. C. Wang, Ed., pp. 387-396, Academic Press, New York, NY, USA, 1979.

[5] M. Mešina, "Convergence acceleration for the iterative solution of the equations $X=A X+f$," Computer Methods in Applied Mechanics and Engineering, vol. 10, no. 2, pp. 165-173, 1977.

[6] C. Brezinski, "Généralisations de la transformation de Shanks, de la table de Padé et de l'epsilon-algorithme," Calcolo, vol. 12, no. 4, pp. 317-360, 1975.

[7] B. P. Pugachev, "Acceleration of the convergence of iterative processes and a method of solving systems of non-linear equations," USSR Computational Mathematics and Mathematical Physics, vol. 17, no. 5, pp. 199-207, 1977.

[8] A. Sidi, W. F. Ford, and D. A. Smith, "Acceleration of convergence of vector sequences," SIAM Journal on Numerical Analysis, vol. 23, no. 1, pp. 178-196, 1986.

[9] P. Wynn, "On a device for computing the em(sn) transformation," Mathematical Tables and Other Aids to Computation, vol. 10, pp. 91-96, 1956.

[10] P. Wynn, "Acceleration techniques for iterated vector and matrix problems," Mathematics of Computation, vol. 16, pp. 301-322, 1962.

[11] C. Brezinski and M. Redivo Zaglia, Extrapolation Methods: Theory and practice, North-Holland, Amsterdam, The Netherlands, 1991.

[12] K. Jbilou and H. Sadok, "Vector extrapolation methods. Applications and numerical comparison," Journal of Computational and Applied Mathematics, vol. 122, no. 1-2, pp. 149-165, 2000.

[13] R. F. King, "A family of fourth order methods for nonlinear equations," SIAM Journal on Numerical Analysis, vol. 10, pp. 876-879, 1973.

[14] L. B. Rall, Computational Solution of Nonlinear Operator Equations, Robert E. Krieger, New York, NY, USA, 1979.

[15] V. Candela and A. Marquina, "Recurrence relations for rational cubic methods: I. The Halley method," Computing, vol. 44, no. 2, pp. 169-184, 1990.

[16] J. He, J. Wang, and J.-C. Yao, "Local convergence of Newton's method on Lie groups and uniqueness balls," Abstract and Applied Analysis, vol. 2013, Article ID 367161, 9 pages, 2013.

[17] V. Candela and A. Marquina, "Recurrence relations for rational cubic methods. II. The Chebyshev method," Computing, vol. 45, no. 4, pp. 355-367, 1990. 
[18] J. M. Gutiérrez and M. A. Hernández, "Recurrence relations for the super-Halley method," Computers \& Mathematics with Applications, vol. 36, no. 7, pp. 1-8, 1998.

[19] Y. Ling, X. Xu, and S. Yu, "Convergence behavior for NewtonSteffensen's method under $\gamma$-condition of second derivative," Abstract and Applied Analysis, vol. 2013, Article ID 682167, 11 pages, 2013.

[20] M. A. Hernández, "Chebyshev's approximation algorithms and applications," Computers and Mathematics with Applications, vol. 41, no. 3-4, pp. 433-445, 2001.

[21] J. A. Ezquerro and M. A. Hernández, "Recurrence relations for Chebyshev-type methods," Applied Mathematics and Optimization, vol. 41, no. 2, pp. 227-236, 2000.

[22] S. Amat, S. Busquier, and Á. A. Magreñán, "Reducing chaos and bifurcations in Newton-type methods," Abstract and Applied Analysis, vol. 2013, Article ID 726701, 10 pages, 2013.

[23] X. Wang, J. Kou, and C. Gu, "Semilocal convergence of a class of modified super-Halley methods in Banach spaces," Journal of Optimization Theory and Applications, vol. 153, no. 3, pp. 779793, 2012.

[24] P. D. Proinov, "New general convergence theory for iterative processes and its applications to Newton-Kantorovich type theorems," Journal of Complexity, vol. 26, no. 1, pp. 3-42, 2010.

[25] X. Xu, Y. Xiao, and T. Liu, "Semilocal convergence analysis for inexact Newton method under weak condition," Abstract and Applied Analysis, vol. 2012, Article ID 982925, 13 pages, 2012.

[26] S. Amat, Á. A. Magreñán, and N. Romero, "On a two-step relaxed Newton-type method," Applied Mathematics and Computation, vol. 219, no. 24, pp. 11341-11347, 2013.

[27] I. K. Argyros and S. Hilout, "Improved generalized differentiability conditions for Newton-like methods," Journal of Complexity, vol. 26, no. 3, pp. 316-333, 2010.

[28] J. M. Gutiérrez, Á. A. Magreñán, and N. Romero, "On the semilocal convergence of Newton-Kantorovich method under center-Lipschitz conditions," Applied Mathematics and Computation, vol. 221, pp. 79-88, 2013.

[29] R. Lin, Y. Zhao, Z. Šmarda, Y. Khan, and Q. Wu, "NewtonKantorovich and Smale uniform type convergence theorem for a deformed Newton method in Banach spaces," Abstract and Applied Analysis, vol. 2013, Article ID 923898, 8 pages, 2013.

[30] J. Kou, S. Sun, and B. Yu, "Multiscale time-splitting strategy for multiscale multiphysics processes of two-phase flow in fractured media," Journal of Applied Mathematics, vol. 2011, Article ID 861905, 24 pages, 2011. 


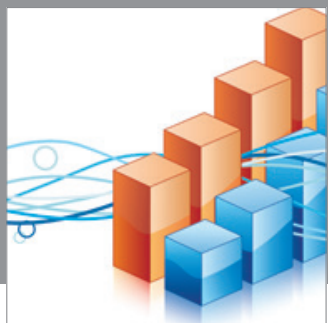

Advances in

Operations Research

mansans

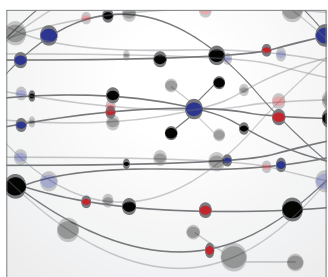

The Scientific World Journal
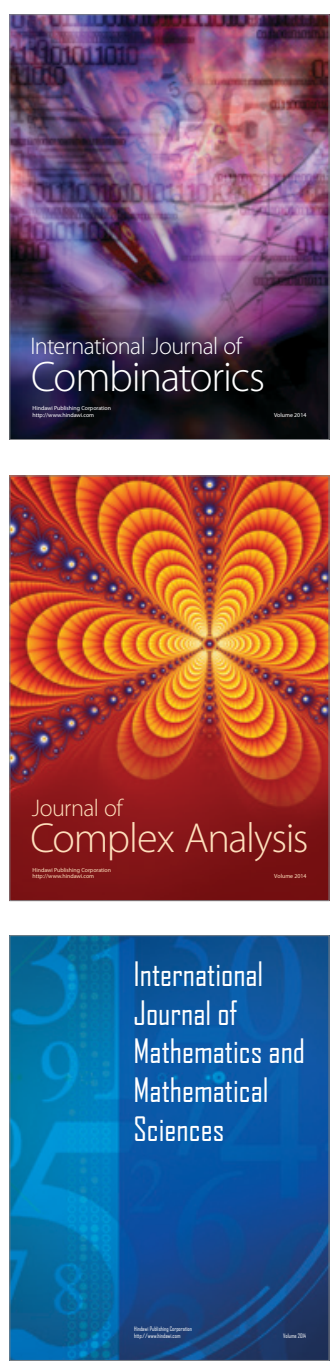
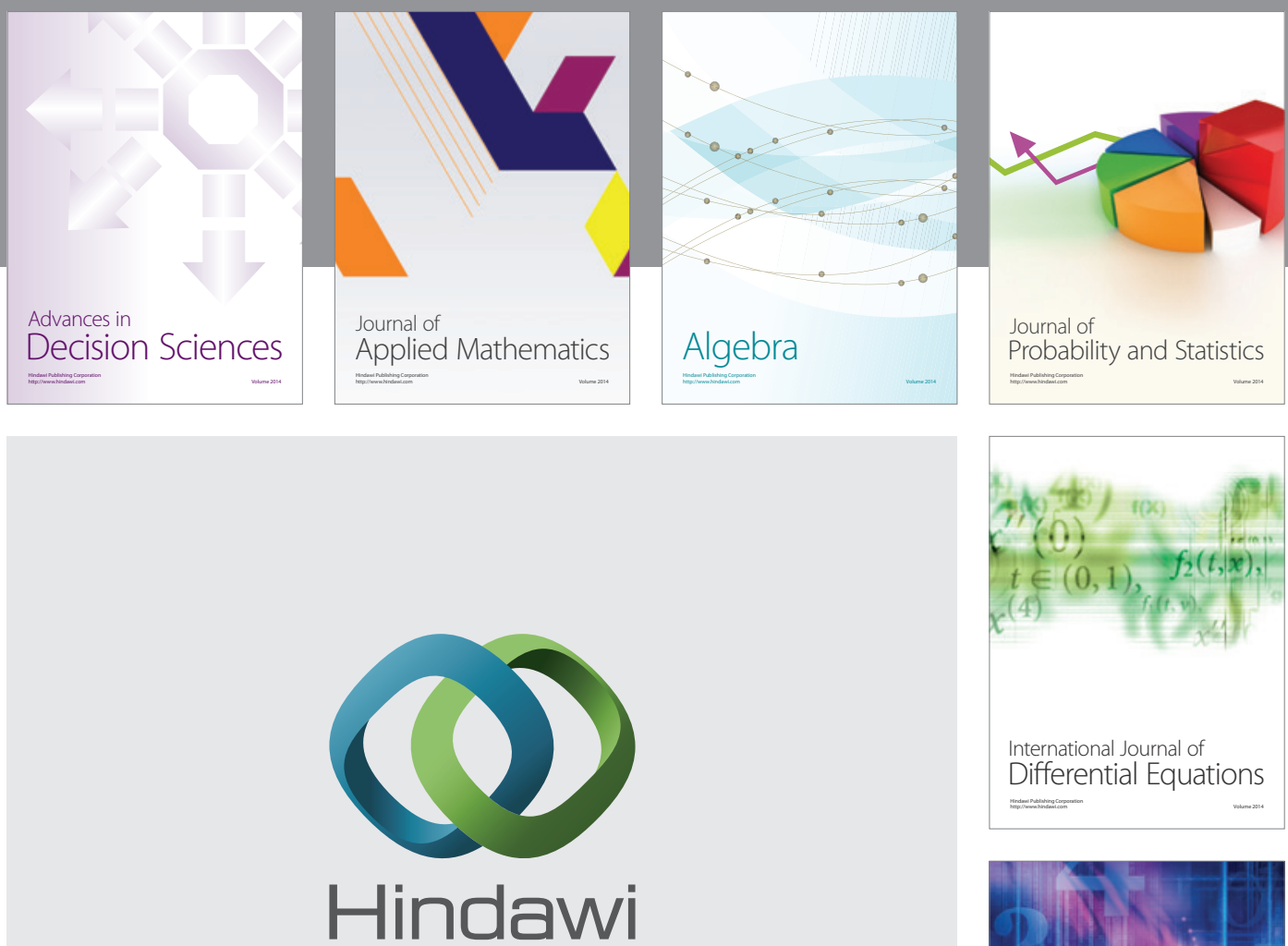

Submit your manuscripts at http://www.hindawi.com
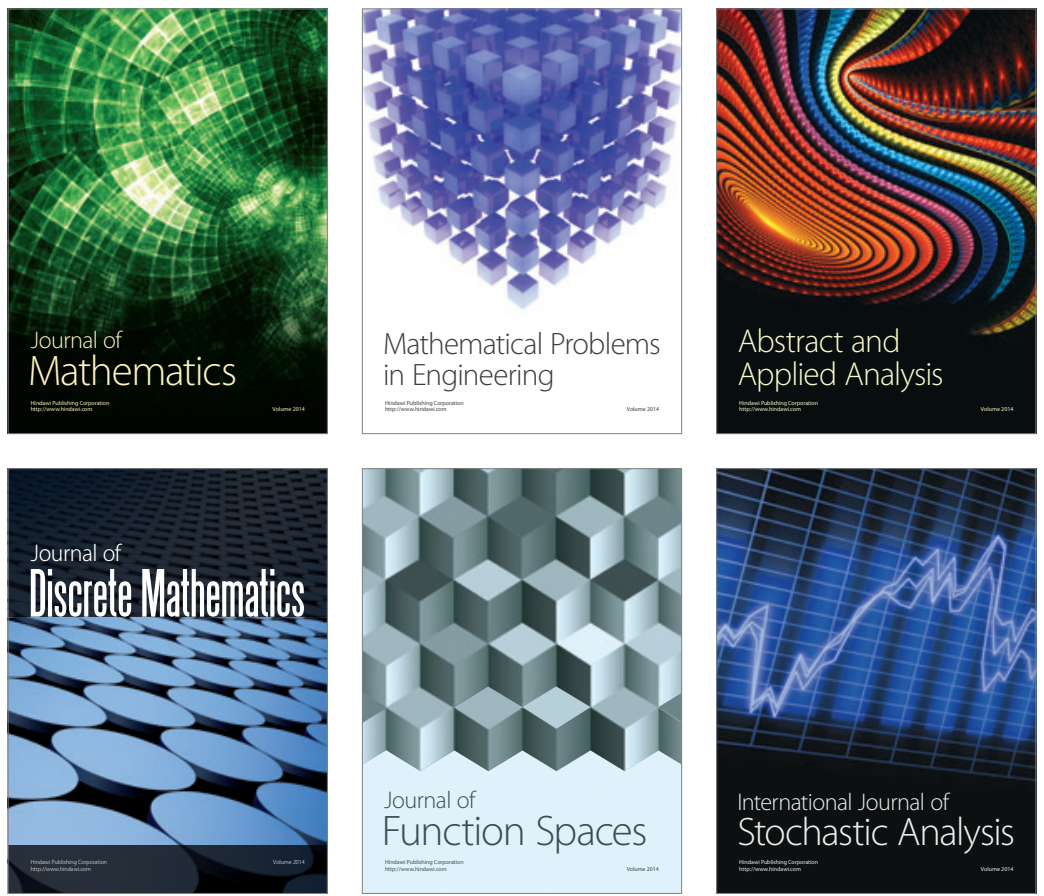

Journal of

Function Spaces

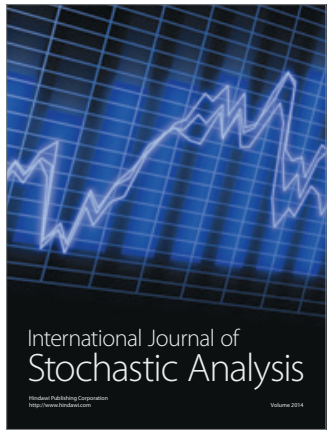

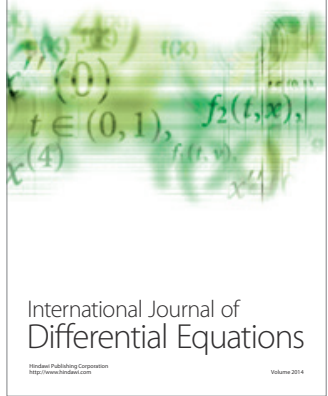
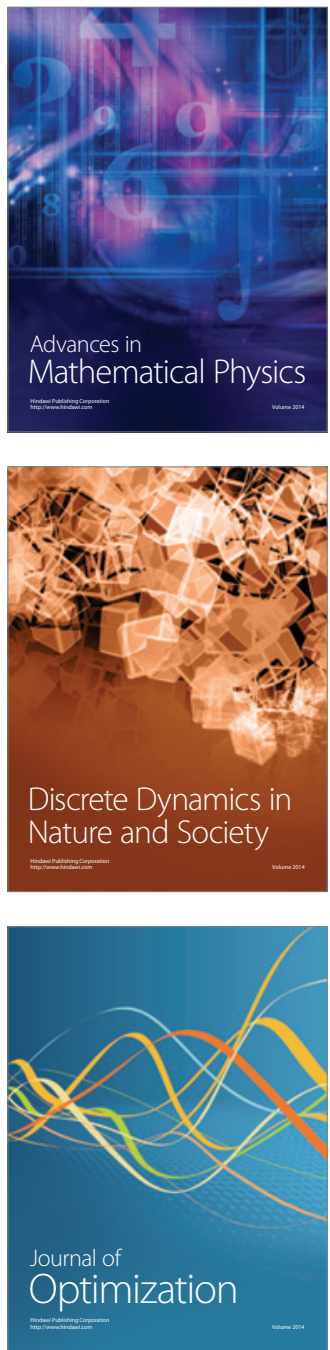